\title{
A Classification Taxonomy and Empirical Analysis of Work Arrangements
}

\author{
Aswani Yeraguntla and Chandra R. Bhat \\ The University of Texas at Austin \\ Department of Civil Engineering \\ 1 University Station C1761 \\ Austin, TX 78712-0278 \\ Phone: (512) 471-4535 \\ Fax: (512) 475-8744 \\ Email: yak@mail.utexas.edu \\ bhat@mail.utexas.edu
}

TRB 2005: For Presentation and Publication

Paper \# 05-1522

Final Submission: April 1, 2005

Word Count: 8,524 


\begin{abstract}
The work-related characteristics of an individual act as pegs around which other non-work activities of an individual are scheduled on a day-to-day basis. In this paper, we identify four dimensions of the medium-to-long range work arrangements that are important to day-to-day temporal and spatial characteristics of work patterns (and hence to day-to-day activity-travel schedules). The four dimensions are: (1) Full-time versus part-time employment, (2) Teleworking or not, (3) Inflexible or flexible work schedules, and (4) Regular or alternate shifts. The paper develops a comprehensive taxonomy of work arrangements in each of these four dimensions and empirically analyses the factors impacting the work arrangement decisions along three of these dimensions.
\end{abstract}




\section{INTRODUCTION}

Activity-based analysis has received much attention and seen considerable progress in the past two decades (1-3). The activity-based analysis approach to travel demand modeling is based on the notion that travel is derived from the need to participate in activities distributed over time and space. An important component of such an activity-based analysis approach is the generation of the number and type of activity episodes undertaken by individuals (i.e., individual activity program generation).

A conceptual framework for the individual activity program generation process was developed by Bhat and Koppelman (4). The basic principle behind this framework for employed individuals is that the work activity acts as a peg around which other non-work activity episodes are scheduled and pursued. The concept that the work activity determines the non-work activity participation characteristics of employed individuals is retained in one form or the other in almost all empirical activity-based modeling studies to date (2, 5-8). However, there have been few studies in the transportation field or in other fields focusing on modeling the many dimensions of work activity arrangement. While one could argue that work-related decisions are dictated by employers, this misses the point that individuals make career decisions over their lifetime and switch jobs in the medium-to-long term to locate a job with their preferred work arrangement (4). Besides, there is evidence that employers are increasingly providing more flexibility in work arrangements to conform to the diverse career paths and balance individuals may desire $(9,10)$.

To summarize, work-related attributes have a substantial impact on the activity-travel choices on a day-to-day basis. At the same time, individuals make medium-to-long term decisions about their work paths based on their preferences. In the current paper, we develop a taxonomy to characterize the many different kinds of work arrangements existing in the work environment today. To focus the scope of our enquiry, we only examine the work arrangements of the vast majority of workers who travel to a distinct out-of-home work location on one or more days of the work week. We will refer to such workers as non-home-based workers, as opposed to home-based workers who work completely from within their homes (such as those individuals involved in home-based businesses). The Current Population Survey of 2001 indicates that about 95\% of the work force may be categorized as non-home-based workers (11). Within the group of non-home-based workers, we further confine our attention to those who have a well-established regular workplace (regular workers) as opposed to those who do not have a regular workplace (these are non-regular workers, such as on-call workers, temporary help agency workers, and self-employed individuals working at a client's location and without a regular work location; see Cohany, 12). In addition to developing a taxonomy of the work arrangements of regular, non-home-based, workers, we also estimate models for three important dimensions characterizing the work arrangements of such workers (for conciseness, we will use the terms "workers" and "work arrangements" in the rest of the paper to refer to regular, nonhome-based, workers and the work arrangements of regular, non-home-based workers, respectively).

The rest of this paper is organized as follows. Section 2 provides an overview of the dimensions characterizing the work arrangements of individuals. Section 3 discusses the work arrangement dimensions examined empirically in the current study, identifies the model structure used for each of the dimensions, and provides a brief overview of earlier empirical studies along each dimension. Section 4 describes the preparation and assembly of data for model estimation. 
Section 5 presents the empirical results. Finally, Section 6 summarizes the study findings and discusses future extensions of the current research.

\section{TAXONOMY FOR WORK ARRANGEMENTS}

There are four primary dimensions that characterize work arrangements: (1) Full-time or parttime employment, (2) Teleworking or not, (3) Inflexible or flexible work schedule, and (4) Regular shift or an alternate shift. These primary dimensions influence the spatial and temporal aspects of the commute. Specifically, the full-time work, no teleworking, inflexible work schedule, and regular shift combination is the "traditional" work arrangement generating the peak temporal concentration of commute trips along high residential density to high employment density spatial corridors. The part-time work, teleworking, flexible schedule and alternate shift combination is the more uncommon arrangement, though the work habits of individuals is increasingly exhibiting one or more of these unconventional characteristics. In the next four sections, we discuss the many facets of each of the four dimensions identified above in the context of the uncommon alternative within each dimension. Thus, the next section discusses a taxonomy for part-time employment characteristics, Section 2.2 for teleworking, Section 2.3 for flexible work schedules and Section 2.4 for alternate work shifts.

\subsection{Part-Time Employment}

Workers with work hours less than 34 hours are referred to as part-time employees in the United States (13). Part-time employees can be classified into three categories: regular part-time employees, employees involved in job sharing, and moonlighters.

\subsubsection{Regular Part-Time Employees}

Individuals who are on an organization's payroll, and who work less than a full work week and who are not short-term hires, are referred to as regular part-time workers. Regular part-time workers are mainly used by organizations to provide assistance during peak time periods of the day or week. Workers' constraints associated with not able to work full-time (due to such reasons as being a student or having child) and employers' difficulty in finding quality full-time employees are the other reasons for the increasingly widespread adoption of regular part-time employment (14).

\subsubsection{Job Sharing}

Job sharing is a more recent work arrangement where a full-time job is shared by multiple (mostly two) individuals. Responsibilities associated with the job, and in some cases salaries and perks, are shared among the individuals. An important aspect of a job sharing work arrangement is the close and open communication between the individuals involved. Individuals who prefer challenging jobs (without the low key, routine, nature of many part-time jobs), and who are unable to work full time because of other responsibilities, are likely to find job sharing work arrangements appealing. Challenges with such job sharing arrangements from an employer standpoint include the more complex paperwork involved in work contracts, and potential productivity losses if the individuals in such arrangements are incompatible in working styles.

Three variations of job sharing arrangements, based on work timing, can be identified split day, split week, and alternate weeks. The adoption of a particular arrangement from among these three different types of job sharing arrangement is primarily based on the work being handled and the availability of individuals who are involved in the job-sharing (15). In a split day 
arrangement, the employees in the job sharing arrangement visit the work place every working day with either a small window of overlapping time (to hand over responsibilities) or nonoverlapping work hours. Here the important work-related attributes are the work start time, work end time and work hours of individuals. In a split-week schedule, the number of days worked is split among the individuals in the job sharing arrangement. The most common split week schemes when there are two individuals in the job sharing arrangement are two and half days each, and two days +3 days with each individual working two days one week and three days the next. Finally, an arrangement where each employee works every other alternate week is referred to as the alternate week job sharing arrangement.

\subsubsection{Moonlighters}

Moonlighters are individuals holding multiple jobs or businesses, which may be all part-time jobs/businesses or a combination of full-time and part-time jobs/businesses. The primary reason for moonlighting is likely to be low wages associated with the main job/business. Other reasons can include the motivation to learn new skills, and the preparation for a career change. The jobs/businesses most often associated with moonlighting are in services, entertainment, public administration and college teaching $(16,17)$.

\subsection{Teleworking}

Advances in telecommunications and information technologies have changed the work patterns of individuals, through the evolution of the teleworking option. Teleworking is defined as the use of communication technologies to pursue business at or from a distance, and includes working away from the regular workplace, mobile work, work at a customer's or client's work location, video-conferencing, telephone conversations, e-mail exchanges, and on-line data base searches (18). The focus in the current paper, however, is on telework that entails working away from the regular workplace in a way that avoids commuting to the regular workplace or impacts the timing of the commute trip to/from the regular workplace (the word "telework" will be interpreted to mean this specific form of telework in the rest of this paper). The potential of teleworking to mitigate urban traffic congestion, reduce energy consumption, and decrease vehicle emissions has led to its widespread promotion as a work option by many transportation planners and policy-makers. From the individual and household perspective, teleworking can increase work schedule flexibility and autonomy and improve job satisfaction and bonds with the family. On the other hand teleworking may also have certain disadvantages, such as generation of new travel patterns (increase in trips for non-work activities), professional and social isolation, and household distractions (19-21).

Several different taxonomics may be used to describe teleworking. In this paper, we develop classification schemes based on the location of telework, the extent of teleworking and the communication protocols involved. Each of these is discussed in turn in the next few sections.

\subsubsection{Work-Location Based Classification}

An individual may telework from home, from a regional center, or a combination of home and a regional center.

Home-based teleworking corresponds to the situation where the individual works from home on one or more days of the work week or the work month. Home-based teleworking avoids commute trips on one or more days of the week/month. It can also boost the productivity of an 
employee, though some individuals may be more productive at work. Home-based teleworking, though it reduces commute trips, may generate new trips and modify the distribution of trips over space and time $(20,21)$.

Regional center-based teleworking corresponds to the case where the employee works at a regional office nearer to home rather than commuting to the main office $(22,23)$. Regional center-based teleworking decreases the commute distance and time and can also potentially reduce the mental stress associated with travel in traffic. Regional center-based teleworking changes travel patterns by substituting the usual commute pattern with travel to the regional office, and changing the number and spatial-temporal characteristics of non-work activity pursuits. Regional center-based teleworking itself may be of one of three types, based on the proprietorship of the regional office. The regional center can be a satellite center owned and operated by a single firm with the goal being to reduce the commute time of employees, or a local center owned and operated by several public and/or private organizations, or a neighborhood center specifically targeted toward the workforce within walking distance of a neighborhood $(22,23)$.

Finally, combination teleworking corresponds to the case when an individual combines home-based teleworking with regional center-based teleworking.

\subsubsection{Extent of Teleworking}

A second classification scheme for teleworking is based on the extent of teleworking. An employee may work almost full-time from home or from a regional office, only very occasionally commuting to the main office for meetings with other colleagues or to report to the employer. We refer to such an arrangement as "full-time teleworking" in this paper. Alternatively, and much more commonly, the employee may regularly work in the main office, while working occasionally out of home or a regional office. We refer to such an arrangement as "part-time teleworking”. Many employees may perceive part-time teleworking as a way to maintain professional and social standing among peers, while also availing the benefits of reduced commute time. Employers may view part-time teleworking as a way to boost employee productivity, while maintaining some control over the work performance of employees.

Within part-time teleworking, there may be two variants: teleworking on one or more days over a certain time period, and teleworking for part of a day. While there has been a reasonable amount of literature on the former kind of part-time teleworking, there has been very little examination of the extent and nature of the latter type of part-time teleworking (i.e., teleworking for part of a day). This latter arrangement can still provide transportation benefits if employees are able to be at their main workplace at times that do not entail commuting during the peak congestion periods.

\subsubsection{Communication Protocols}

Another classification of teleworking is based on the form of communication between a teleworker and his/her colleagues and employer. Some teleworkers are required to stay electronically connected during business hours. Such a protocol arrangement can have a significant impact on the ability to undertake non-work activities at one's desired time and choosing. For many teleworkers, communication is through e-mails, phone conversations and periodical video-conference meetings (24). 


\subsection{Flexible Schedule}

A flexible schedule is one where an employee selects his/her work schedule on a day-to-day basis or on a periodic basis, but is required to work a certain number of hours at the work place during a given period. Flexible schedules provide an opportunity for employees to plan their work timings or work days so that they can avoid peak period congestion and reduce the conflict between their work and non-work responsibilities. The increasing adoption of the flexible scheduling work option both by employers and employees is evident from the May 2001 Current Population Survey. According to this survey, 28.8\% of full-time workers have flexibility to vary their work start and end times, nearly double the percentage in 1991 (25).

The flexible work scheduling options include flexitour, gliding schedule, flexitime, and compensatory time (see also 26, 10). Each of these options is briefly discussed in turn below.

\subsubsection{Flexitour}

Flexitour represents the lowest degree of flexibility in work arrangements. In such a work arrangement, a worker is required to work a specified number of hours each work day, but is allowed to choose arrival and departure times around an established set of mandatory "core hours". Once chosen, however, the work hours become fixed for an employee over a period. Core hours are the work hours during which every employee in the organization should be at the work place. Once an employee chooses a schedule, his/her daily work attributes are fixed over a period. But within an organization, the work start time and end times of employees could vary.

\subsubsection{Gliding Schedule}

Gliding schedule is the next level in the hierarchy of flexibility. As in the flexitour arrangement, the employee is scheduled to work for a specified number of hours each day and can choose the work start and end times around an established set of mandatory "core hours". However, unlike the flexitour arrangement, the employee can vary his/her work timing each work day. Thus, work start and end times might vary not only across employees of the same cadre, but also across work days of an employee.

\subsubsection{Flexitime}

The highest level of flexibility is offered by the flexitime work arrangement. In this arrangement, employees can vary their work timings and work hours at their choice, but have to work a specified number of hours during the pay period. Three categories of flexitime work arrangements, based on work timing and work hours, may be identified: (1) variable day schedule, (2) variable week schedule, and (3) maxiflex schedule. In the variable day schedule, an employee works every day, but is allowed to vary the number of hours worked each day. Hence, work start time and end times, and work hours, are likely to vary across work days. The variable week schedule allows employees to vary the length of their work week as well as the number of hours worked each day. Hence, in addition to the work timing and number of hours worked each day, the number of days an employee commutes to work is also an important variable in this work arrangement. But, once chosen, the number of work days in the work week (or pay period) is fixed. The maxiflex schedule allows an employee to vary the number of hours worked each day and number of days worked each week. As opposed to the variable-week schedule, the number of days worked each week is not fixed. 


\subsubsection{Compensatory Time Arrangement}

In compensatory time work arrangements, employees work overtime during some days of the work week. Instead of getting paid for overtime, employees accumulate the extra hours for future time-off.

\subsection{Alternate Shifts}

Work arrangements with work timings between $6 \mathrm{PM}$ in the evening of one day and $8 \mathrm{AM}$ in the morning of the next day are grouped under the category of alternate shifts. The nature of work is a major determinant of whether an individual has an alternate shift. Specifically, individuals who are police officers, emergency room physicians and assembly-line workers at a factory are likely to be the kind of individuals with alternate shifts. According to the May 2001 Current Population Survey, 14.5 percent of all full-time wage and salary workers work in alternate shifts (25).

The most prevalent alternate shifts are evening shift, night shift, rotating shift and irregular shift (27). The evening shift, in general, starts after 2 PM and ends before midnight. The night shift refers to arrangements with work hours between 9 PM and 8 AM. A rotating shift corresponds to the case when work hours change periodically from days to evenings or nights and vice-versa. Irregular shifts are associated with work hours that change without any kind of periodicity.

\section{EMPIRICAL ANALYSIS FOCUS AND MODELING STRUCTURES USED}

The previous section developed a taxonomy for "atypical" work arrangements defined by parttime employment, teleworking, flexible work schedules, and alternate shifts. In this paper, we focus on an analysis of the first three dimensions identified above. We do not model the alternate shift dimension because of lack of adequate individuals working alternate shifts in the sample used in our analysis (we use a web-based survey of Austin area commuters conducted between December 2003 and January 2004 for the analysis; details of this data are provided in the next section).

\subsection{Part-Time Employment}

The first dimension, part-time employment, is modeled using a simple binary choice model for the choice between part-time and full-time employment. Part-time employment is quite widespread and is expected to grow further in the United States because of the reduced availability of full-time jobs (28). We are unable to further model the breakdown of part-time employment by regular part-time employment, job sharing, and moonlighting because of the inability to make such distinctions from the 2004 Austin commute survey. However, unlike most earlier studies of part-time employment in non-transportation fields that confine their attention to women (29-31), we examine part-time employment choice in the overall worker population.

\subsection{Teleworking}

The second dimension, teleworking, is modeled using a binary choice model for the decision to telework and an ordered response model for the extent of teleworking. Due to data limitations, we are unable to model the location and communication protocol aspects of teleworking arrangement; the focus is on whether or not an individual teleworks from home and the extent (frequency) of this teleworking. Further, we confine attention on teleworking from home instead of commuting to the work place. We do not address the case of individuals teleworking from 
home for part of the day and working at their regular workplace for part of the day because of data unavailability on this issue.

The binary decision to telework and the ordered response decision of the extent of teleworking are jointly modeled by allowing the error terms in the two decisions to be correlated to account for common unobserved characteristics affecting these two decisions. For example, individuals who are techno-savy or are environmentally conscious may be the ones who choose to telework and also telework frequently (see Popuri and Bhat, 21, for the model structure and estimation details of such a joint binary choice-ordered response formulation).

There is an extensive body of modeling literature on the narrow view of teleworking adopted in this study (i.e., teleworking from home instead of commuting to the work place). Specifically, several researchers have used stated preference surveys to examine the impact of demographic, occupational, and attitudinal factors on teleworking choice (32-36). In addition, to better quantify the level of teleworking, while also examining the impact of relevant factors on teleworking levels, several studies have used revealed preference surveys (37-39, 21). The current study contributes to this existing literature by using a revealed preference survey. The methodology is similar to the one used in Popuri and Bhat, though our study also considers teleworkers who work from home less frequently than once a week. Popuri and Bhat's study did not consider such teleworkers, though a significant fraction of teleworkers are likely to work from home less than once a week (51\% of teleworkers in the sample used in the current study telework less often than once a week).

\subsection{Flexible Schedule}

The third dimension, flexible schedule, can correspond to many different options, including flexitour, gliding schedule, flexitime, and compensatory time arrangement. However, data on work arrangements are seldom collected in today's data sets at a level of detail that enables us to identify the type of flexible schedule. In the current paper, we use a proxy measure as an indicator of flexible schedules. Specifically, the survey asks individuals to provide their usual work timings and the ease with which they can arrive at work 15-30 minutes late from a work scheduling standpoint. This ease of arriving late is used as a measure of flexibility in schedule. Admittedly, this measure combines multiple aspects of work schedules. For example, the measure may be the leeway in schedule offered to workers with otherwise inflexible schedules, or the flexibility in arriving late for workers with a flexitour work arrangement (see Section 2.3.1), or simply an indication of the presence of a gliding schedule (Section 2.3.2). Future research should collect more specific information regarding flexible work schedule arrangements to clearly disentangle the many possibilities identified above.

A rating scale was used in the survey to capture the measure of the ease of arriving 15-30 minutes late (see next section for further details) and so an ordered response model was developed for the flexible schedule dimension of the analysis.

Our review of the literature indicates little empirical research into flexible schedules. There have been a few studies in labor economics oriented toward understanding what workers trade off to get flexible schedules (10), or to examine wage differentials between flexitime and regular work schedules (40), or case studies of businesses that offer some kind of flexible work schedules (41). However, except for Golden, none of these earlier studies examine the factors impacting the propensity to have a flexible work schedule. 


\section{DATA SOURCE}

The primary data used in the current analysis is drawn from a web-based survey of Austin area commuters. In addition to this primary data source, we also obtained secondary zonal land-use and zone-to-zone network skim-distances from the Capital Area Metropolitan Planning Organization (CAMPO). In the rest of this section, we focus on a description of the primary data source; the web-based commuter survey; used in the analysis.

\subsection{Survey Administration}

The survey was administered through a web site hosted by the University of Texas at Austin (see http://www.ce.utexas.edu/commutersurvey/index.htm). The survey was designed for the internet using a combination of HTML code and Java. Further details of the survey design and survey administration are available in Bhat and Sardesai (42).

\subsection{Sample Formation and Description}

The data from the completed web surveys were downloaded in ASCII format, and then imported into SPSS to label and code the variables appropriately. The final sample included between 657 and 699 individuals, depending on the dimension of analysis (this is because some individuals did not provide adequate information on some work arrangement dimensions). The next three paragraphs provide details on the sample size and choice splits for each analysis dimension.

For the part-time versus full-time employment analysis, the sample size was 699 workers, with 66 (9.4\%) workers in part-time employment and 633 (90.6\%) workers in full-time employment.

The teleworking choice and frequency measures were obtained from the survey by presenting the following questions: "Do you currently work from home on some weekdays instead of traveling to your regular workplace outside home?" and "How often do you work from home instead of traveling to your regular workplace outside home?" The sample size for the teleworking dimension analysis comprised 687 individuals, of whom 82 (12\%) workers reported teleworking from home on some weekdays. Within the sample of 82 teleworkers, 24 (3.5\% of overall sample) teleworked once a month, 5 (0.7\%) teleworked once every 3 weeks, 13 (1.9\%) teleworked once every 2 weeks, 19 (2.8\%) teleworked once a week, 17 (2.5\%) teleworked 2-3 times a week and $4(0.6 \%)$ teleworked 4 or more times a week.

Flexibility, as represented in this study, is the ease with which an employee can arrive 3045 minutes late at work, as indicated in Section 3.3. A measure of this ease was obtained from respondents by presenting them the following question, "From a work schedule perspective, how easy would it be for you to arrive at work in the morning 30-45 minutes late”. Individuals were asked to respond on a 5 point scale ranging from 1 (very difficult) to 5 (very easy). The sample size for this analysis was 657 workers, with the number (percentage) of workers answering at each level of the scale being as follows: 1 (very difficult)- 256 (39\%), 2- 97 (14.8\%), 3- 98 (14.9\%), 4- 66 (10\%), and 5 (very easy)- 140 (21.3\%). This indicates a reasonable distribution along the flexible schedule scale.

\section{EMPIRICAL RESULTS}

\subsection{Explanatory Variables}

We considered several types of explanatory variables in the models estimated in the paper. These variables can be broadly classified into four categories: (1) individual sociodemographics, (2) household characteristics, (3) land use characteristics of the individual's main workplace, and (4) 
work characteristics. In our specification analysis, we used a t-statistic value of 1.00 as the threshold for retaining variables due to the relatively small sample size of commuters and the very small fraction of workers who hold part-time jobs and who telework.

\subsection{Part-Time Versus Full-Time Employment}

A binary logit model was used to model part-time vs. full-time employment. The final specification of the model is presented in Table 1 and discussed below by variable category. The parameters represent the impact on the propensity to be employed part-time.

\subsubsection{Effect of Individual Sociodemographics}

The only individual sociodemographic variable that turned out to be significant was age (however, see also the interaction effect of being a female and having children in the household, discussed under household characteristics). The age effect indicates that young adults (less than 25 years) are more likely to be in part-time employment than older adults. This is perhaps a reflection of the fact that many young adults are studying and working part-time at the same time ${ }^{1}$.

\subsubsection{Effect of Household Characteristics}

The impact of household characteristics in Table 1 shows that women in households with children are particularly likely to be part-timers, reinforcing the notion that women are the primary caregivers of children (see Miller, 29, who also found a similar result). Further, individuals in households with several vehicles are less likely to be part-timers compared to individuals in households with few vehicles.

\subsubsection{Effect of Work-Related Attributes}

The results show that employees working for an educational institution are more likely to be part-timers than employees in other kinds of organizations. This is likely to be because of graduate students, who work part-time and are associated with Universities in and around Austin. Finally, the effect of the length of service shows that employees who have worked less than a year in the firm are more likely to be part-timers than those who have been working for longer periods of time. Of course, this effect may also be simply a result of the more temporary nature of many part-time employment jobs.

\subsubsection{Measures of Fit}

The measures of fit for the binary choice model are provided toward the bottom of Table 1 and are self-explanatory. The likelihood ratio statistic for testing if the independent variables in the model aid in predicting the employment status of an individual is 81.9, which is larger than the critical chi-squared table value with 5 degrees of freedom at any reasonable level of significance.

\subsection{Teleworking}

A joint binary choice-ordered Response Probit (ORP) model is used to analyze teleworking choice and the extent of teleworking. Teleworking choice is a binary response variable: an individual either teleworks or not. The extent of teleworking is represented in the analysis in terms of 6 ordinal categories: (1) telework once a month, (2) telework once every 3 weeks, (3)

\footnotetext{
1 The reader will note that we did not have a separate question on student status in the survey, and so could not include student status directly in the model.
} 
telework once every 2 weeks, (4) telework once every week, (5) telework 2-3 times a week, and (6) telework 4 or more times a week. Table 2 presents the parameter estimates of the final preferred specification by variable group.

\subsubsection{Effect of Individual Sociodemographics}

The results indicate that women adults in households without children are less likely to telework than male adults in households without children (though this effect is not statistically significant even at our generous t-statistic value of 1.00). However, women adults in households with children are more likely to telework than men adults in households with children. The first effect above may be a reflection of the overrepresentation of men in telework-friendly occupations, while the second effect is perhaps due to women being the primary caregivers of children. The latter effect has also been reported by Drucker and Khattak (39) and Popuri and Bhat (21).

The effects of race in Table 2 are not immediately intuitive. They indicate that Caucasians and Hispanics are more likely to telework than other races (African-Americans, Asians and other). However, within the group of individuals who telework, individuals of Caucasian and Hispanic races telework less often than individuals of other races.

Finally, within the group of individual sociodemographics, individuals with an undergraduate degree or higher, and employees not in the low income bracket, appear to be more likely to telework, suggesting that either highly educated and not-low-income earning individuals are able to exercise more independence and control over their work schedule, or are the ones more likely to be in jobs conducive to teleworking. These results are consistent with the findings of Bagley and Mokhtarian (36).

\subsubsection{Effect of Household Characteristics}

There was only one household variable; number of household vehicles; that turned out to be statistically significant in impacting teleworking behavior. The impact of this variable indicates that individuals with several vehicles in their household are less likely to telework, perhaps because they do not face the kind of mobility constraints encountered by individuals with fewer vehicles in their household (Yen 43, notes a similar finding). An alternative explanation is that individuals with several vehicles may be more likely to have a nice and/or new car, and may associate a certain amount of status to commuting to work by their nice/new car.

\subsubsection{Effect of Work-Related Variables}

The individual work-related variables indicate that part-time employees are more likely to telework and telework frequently relative to full-time employees (a similar result was observed by Drucker and Khattak 39, and Popuri and Bhat 21). This is presumably because the familial and other responsibilities that influence an individual to seek part-time employment also influence the individual to pursue jobs that provide teleworking opportunities.

The nature of the employing company also has an impact on the choice to telework and the extent of teleworking. In particular, individuals working in educational institutions (think professors!) and in federal government offices are more likely to telework than individuals working with other kinds of employers, while individuals working with the state government are less likely to telework. This suggests a more telework-friendly work environment in education institutions and federal government-related jobs (for example, Joice 44 provides an overview of teleworking programs available for federal government employees). 


\subsubsection{Threshold Parameters and Correlation Between Telework Choice and Frequency}

The threshold parameters determine the correspondence between the latent propensity to telework and the observed teleworking frequency level. In the telework choice binary model, the "threshold 1" parameter is estimated in lieu of a constant term. In the telework frequency ordinal model, we estimated five threshold parameters, since we did not include a separate constant term in the latent propensity to telework. As such, the threshold parameters do not have any substantive behavioral interpretation.

The sign of the correlation term in the joint telework choice - telework frequency model (see fourth row from bottom in Table 2) indicates a high and very significant positive correlation in unobserved factors affecting teleworking choice and teleworking frequency. Such unobserved factors may include individual-specific personality traits, such as a desire for enhanced interaction with the family, an intrinsic aversion for the commute, and a preference for a work environment that is isolated from co-workers.

\subsubsection{Measures of Fit}

Several measures of fit are computed for the joint binary choice-ordered response model of telework choice and frequency, as explained in Table 2. The likelihood ratio test value for the null hypothesis that none of the independent variables and the correlation parameter help in explaining telework choice and frequency is 72.6. This is larger than the critical chi-squared table value with 15 degrees of freedom even at the 0.001 level of significance, clearly highlighting the value of the joint model estimated here.

\subsection{Flexible Schedule}

As indicated in section 4, we capture the dimension of "flexible schedule" by the ease of arriving at work 30 to 45 minutes late. A rating scale was used in the survey to capture this measure of "ease". Specifically, respondents were asked to characterize, on a five point scale from "very difficult" at the left extreme to "very easy" on the right extreme, their perceived comfort level in arriving at work in the morning between 30-45 minutes late. The 5-point ordinal scale was used as the dependent variable in the ordered response probit model. The various effects provide the impact of each variable on the propensity to have a flexible schedule. The results are presented in Table 3 and are discussed by variable group below.

\subsubsection{Effect of Individual Sociodemographics}

Three individual sociodemographics were observed to impact work schedule flexibility. The influence of the first variable, age, shows that younger employees (less than 25 years of age) are less likely to have work schedule flexibility relative to employees older than 25 years. A similar result was reported by Golden (10). The effect of the other two variables suggest that high income individuals and employees with an undergraduate degree or higher have a higher likelihood of having more flexibility relative to low income employees and employees who do not have an undergraduate degree, respectively. These findings are consistent with the notion that high income and well-educated individuals are able to exercise more control over their work schedule than are their co-workers.

\subsubsection{Effect of Household Characteristics}

Only one household variable, presence of children less than 5 years in the household, had an impact on work schedule flexibility. The effect of this variable shows that adults in household 
with small children are more likely to be in a flexible work arrangement. This may be a result of employers being more flexible to employees with small children and/or adults in households with small children choosing jobs that provide work schedule flexibility.

\subsubsection{Effect of Work-Related Variables}

The results of work-related variables indicate that individuals starting work earlier than 9 AM in the day have lesser work schedule flexibility than employees who work later (after 9 AM). On the other hand, individuals whose employment locations are in high density urban and CBD areas have higher work schedule flexibility.

\subsubsection{Threshold Parameters}

The threshold parameters determine the correspondence between the latent propensity to have a flexible schedule and the reported flexibility ratings, and do not have any substantive behavioral interpretation.

\subsubsection{Measures of Fit}

The various measures of fit computed in Table 3 are again self-explanatory. The likelihood ratio test for the usefulness of the model is 33.48, which is higher than the chi-squared critical value with 6 degrees of freedom at any reasonable level of significance. Of course, the very low rhobar squared value with respect to the sample shares $(=0.011)$ in Table 3 perhaps also indicates the need to improve the measure of flexibility used, as discussed earlier in Section 3.3.

\section{SUMMARY AND CONCLUSIONS}

The work-related characteristics of an individual act as pegs around which other non-work activities of an individual are scheduled on a day-to-day basis. Most current activity schedule systems, however, do not focus adequate attention on work-related attributes. There have also been few studies in other fields focusing on modeling the many dimensions of work activity arrangements.

In this paper, we identify four dimensions of the medium-to-long range work arrangement decisions of non-home-based workers who have a well-established regular workplace outside home. The four dimensions we identify are important to the day-to-day temporal and spatial characteristics of work patterns (and hence to day-to-day activity-travel schedules), and include: (1) Full-time versus part-time employment, (2) Teleworking or not, (3) Inflexible or flexible work schedules, and (4) Regular or alternate shifts. The paper then proceeds to develop a comprehensive taxonomy of work arrangements in the context of the more uncommon alternatives (i.e., part-time employment, teleworking, flexible schedule and alternate shifts) in the four dimensions.

The empirical analysis in the current study focuses on the modeling of the first three dimensions identified above. The models developed in this paper may be included as precursor medium-term models to a daily activity-travel pattern modeling system. Such a comprehensive modeling system is beneficial for at least three reasons. First, using the work arrangement model to determine the broad working preferences and habits of individuals informs the day-to-day work schedule modeling process. Specifically, a knowledge of the work arrangement of an individual can help better predict the day-to-day work schedule of the individual. To the extent that day-to-day work schedules are critical pegs in daily activity schedule modeling, work arrangement modeling is critical to activity travel pattern modeling. Second, the models 
developed in this paper provide useful information to identify the kinds of individuals and the work occupations that may be most amenable to a change from a "traditional" work arrangement to a more "unconventional" work arrangement. To the extent that the unconventional work arrangements may contribute to traffic congestion alleviation and air quality improvement, the models can be used to inform transportation demand management strategies. Third, the models can be used in a pure predictive mode to accurately predict the impact (on work arrangement and hence individual daily travel patterns) of changing demographics over time.

\section{ACKNOWLEDGEMENTS}

The authors would like to thank Allison Lockwood, Harsha Nerella, and Aruna Sivakumar for help with the web survey design, testing, and data extraction. Several other transportation graduate students also assisted in disseminating information regarding the survey. The authors are indebted to the Clean Air Force of Central Texas (especially Wendy Willingham and Deanna Altenhoff) and Nustats, Inc. (Johanna Zmud and Mia Zmud) for their support of this research effort. Three anonymous reviewers provided valuable comments on an earlier version of the paper. 


\section{REFERENCES}

1. Bhat, C.R., and F.S. Koppelman. A Retrospective and Prospective Survey of Time-Use Research. Transportation, Vol. 26, No. 2, 1999, pp. 119-139.

2. Pendyala, R.M., and K.G. Goulias. Time Use and Activity Perspectives in Travel Behavior Research. Transportation, Vol. 29, No. 1, 2002, pp. 1-4.

3. Arentze, T.A., and H.J.P. Timmermans. A Learning-Based Transportation Oriented Simulation System. Transportation Research Part B, Vol. 38, 2004, pp. 613-633.

4. Bhat, C.R., and F.S. Koppelman. A Conceptual Framework of Individual Activity Program Generation. Transportation Research Part A, Vol. 27, No. 6, 1993, pp. 433-446.

5. Damm, D. Interdependence in Activity Behavior. Transportation Research Record, Vol. 750, 1980, pp. 33-40.

6. Bowman, J.L., and M.E. Ben-Akiva. Activity-Based Disaggregate Travel Demand Model System with Activity Schedules. Transportation Research Part A, Vol. 35, 2000, pp. 1-28.

7. Bhat, C.R., and S. Srinivasan. A Multidimensional Mixed Ordered-Response Model for Analyzing Weekend Activity Participation. Transportation Research Part B, Vol. 39, No. 3, 2005, pp. 255-278.

8. Bhat, C.R., and S.K. Singh. A Comprehensive Daily Activity-Travel Generation Model System for Workers. Transportation Research Part A, Vol. 34, No. 1, 2000, pp. 1-22.

9. Galinsky, E., J.T. Bond, and J. Swanberg. The 1997 Study of the Changing Work Force. Families and Work Institute, New York, 1998.

10. Golden, L. Flexible Work Schedules: What are we trading off to get them? Monthly Labor Review, Bureau of Labor Statistics, U.S. Department of Labor, Vol. 124, No. 3, 2001, 50-67.

11. Bureau of Labor Statistics, 2002a. Work at Home in 2001. http://www.bls.gov/news.release/homey.nr0.htm. Accessed November 12, 2004.

12. Cohany, S.R. Workers in Alternative Employment Arrangements. Monthly Labor Review, Vol. 119, No. 10, 1996, pp. 31-45.

13. Hamermesh, D.S. Workdays, Workhours, and Work Schedules: Evidence for the United States and Germany. W.E. Upjohn Institute for Employment Research, Kalamazoo, Michigan, 1996.

14. Erickcek, G., and S. Houseman. A Report on Temporary, Part-Time and Contract Employment in the United States. W.E. Upjohn Institute for Employment Research, Kalamazoo, Michigan, 1997.

15. Lederman, J. Two People, One Job: Job Sharing Keeps Parents Working and Helps Companies Retain Employees. The New York Times, January 14, 2001, pp. 8.

16. Kimmel, J. Moonlighting in the United States. Employment Research, Vol. 2, No. 1, W.E. Upjohn Institute for Employment Research, Spring 1995.

17. Fawkner, E. Moonlighting's greatest challenge ... How to beat the time crunch. A HomeBased Business Online (AHBBO), 2000. http://www.ahbbo.com/timecrunch.html. Accessed July 25, 2004.

18. Mokhtarian, P.L. A Typology of Relationships Between Telecommunications and Transportation. Transportation Research Part A, Vol. 24, No. 3, 1990, pp. 231-242.

19. Salomon, I. Technological Change and Social Forecasting: the Case of Telecommuting as a Travel Substitute. Transportation Research Part C, Vol. 6, Issues 1-2. 1998, pp. 17-45. 
20. Kurland, N.B., and C.D. Cooper. Manager Control and Employee Isolation in Telecommuting Environments. Journal of High Technology Management Research, Vol. 13, Issue 1, 2002, pp. 107-126.

21. Popuri, Y.D., and C.R. Bhat. On Modeling Choice and Frequency of Home-Based Telecommuting. Transportation Research Record, Vol. 1858, 2003, pp. 55-60.

22. Nilles, J.M. Traffic Reductions by Telecommuting: A Status Review and Selected Bibliography. Transportation Research Part A, Vol. 22, No. 4, 1988, pp. 301-317.

23. Popuri Y.D. On Modeling the Choice and Frequency of Home-Based Telecommuting by Individuals. M.S. Thesis, Department of Civil Engineering, The University of Texas at Austin, Austin, TX, 2001.

24. Mariani, M. Telecommuters. Occupational Outlook Quarterly, Vol. 44, No. 3, Fall 2000, pp. 10-17.

25. Bureau of Labor Statistics, 2002b. Workers on Flexible and Shift Schedules in 2001 Summary. http://www.bls.gov/news.release/flex.nr0.htm. Accessed November 12, 2004.

26. McCampbell, A.S. Benefits Achieved Through Alternative Work Schedules. Human Resource Planning, Vol. 19, No. 3, 1996, pp. 30-37.

27. Beers, T.M. Flexible Schedules and Shift Work: Replacing the '9-to-5' workday? Monthly Labor Review, Bureau of Labor Statistics, U.S. Department of Labor, Vol. 123, No. 6, 2000, pp. 33-40.

28. Krantz, R., M.D. Natale, and T.J. Krolik. The U.S. Labor Market in 2003: Signs of Improvement by year's end. Monthly Labor Review, Bureau of Labor Statistics, U.S. Department of Labor, Vol. 127, No. 3, 2004, pp. 3-29.

29. Miller, C.F. Part-Time Participation Over the Life Cycle Among Married Women Who Work in the Market. Applied Economics, Vol. 25, 1993, pp. 91-99.

30. Lester, B.Y. Part-Time Employment of Married Women in USA: A Cross-Sectional Analysis. American Journal of Economics and Sociology, Vol. 55, No. 1, 1996, pp. 61-71.

31. Connelly, R., and J. Kimmel Marital Status and Full-Time/Part-Time Status in Child Care Choices. Applied Economics, Vol. 35, 2003, pp. 761-777.

32. Bernardino, A.T., Ben-Akiva, M., and I. Salomon. Stated Preference Approach to Modeling the Adoption of Telecommuting. Transportation Research Record 1413, TRB, National Research Council, Washington, D.C., 1993, pp. 22-30.

33. Mahmassani, H.S., Yen, J., Herman, R., and Sullivan, M. A. Employee Attitudes and Stated Preferences Toward Telecommuting: An Exploratory Analysis. Transportation Research Record, Vol. 1413, 1993, pp. 31-41.

34. Sullivan, M.A., H.S. Mahmassani and J-R. Yen. Choice Model of Employee Participation in Telecommuting Under a Cost-Neutral Scenario. Transportation Research Record, Vol. 1413, 1993, pp. 42-48.

35. Mokhtarian, P. L., and I. Salomon. Modeling the Choice of Telecommuting: 3. The Importance of Attitudinal Factors in Behavioral Models. Environment and Planning A, Vol. 28, 1996, pp. 1877-1894.

36. Bagley, M.N., and P.L. Mokhtarian. Analyzing the Preference for Non-Exclusive Forms of Telecommuting: Modeling and Policy Implications. Transportation, Vol. 24, 1997, pp. 203226.

37. Mannering, J.S., and P.L. Mokhtarian. Modeling the Choice of Telecommuting Frequency in California: An Exploratory Analysis. Technological Forecasting and Social Change, Vol. 49, 1995, pp. 49-73. 
38. Belanger, F. Workers’ Propensity to Telecommute: An Empirical Study. Information \& Management, Vol. 35, 1999, pp. 139-153.

39. Drucker, J., and A.J. Khattak. Propensity to Work from Home - Modeling Results from the 1995 Nationwide Personal Transportation Survey. Transportation Research Record, Vol. 1706, 2000, pp. 108-117.

40. Gariety, B.S., and S. Shaffer. Wage Differentials Associated with Flextime. Monthly Labor Review, Bureau of Labor Statistics, U.S. Department of Labor, Vol. 124, No. 3, 2001, 68-75.

41. Dex, S., and F. Scheibl. Flexible and Family Friendly Work Arrangements in UK-Based Small and Medium Enterprises: Business Cases. British Journal of Industrial Relations, Vol. 39, No. 3, 2001, pp. 411-431.

42. Bhat, C.R., and R. Sardesai. On Examining the Impact of Stop-Making and Travel Time Reliability on Commute Mode Choice: An Application to Predict Commuter Rail Transit Mode for Austin, TX. Technical paper, Department of Civil Engineering, The University of Texas at Austin, August 2004.

43. Yen, J. Interpreting Employee Telecommuting Adoption: An Economics Perspective. Transportation, Vol. 27, 2000, pp. 149-164.

44. Joice, W. The Evolution of Telework in the Federal Government, Office of Governmentwide Policy, U.S. General Services Administration, 2000, http://www.gsa.gov/gsa/cm_attachments/GSA_DOCUMENT/EvolutionteleworkPDF_R2Ec9T_0Z5RDZ-i34K-pR.pdf 


\section{LIST OF TABLES}

TABLE 1 Choice Model Results for Part-time Employment

TABLE 2 Empirical Results for Teleworking-related Decisions

TABLE 3 Choice Model Results for Flexible Schedule 
TABLE 1 Choice Model Results for Part-time Employment

\begin{tabular}{|l|c|c|}
\hline Explanatory Variable & Parameter & t-statistic \\
\hline Individual Sociodemographics & 2.113 & 2.784 \\
\hline Age - less than 25 years & & \\
\hline Household Characteristics & 1.159 & 3.373 \\
Females with presence of children (less than 15 years) & -0.375 & -2.047 \\
\hline Number of vehicles & & 1.927 \\
\hline Work-related Variables & 1.267 & 4.073 \\
\hline Employer is educational institution & -1.250 & -2.955 \\
\hline Length of Service in the firm - less than a year & & 699 \\
\hline Constant & & -484.51 \\
\hline Number of observations & -218.54 \\
\hline Log-likelihood at equal shares $-L(0)$ & -177.59 \\
\hline Log-likelihood at sample shares $-L(c)$ & 0.621 \\
\hline Log-likelihood at convergence $-L(\hat{\beta})$ & & 0.165 \\
\hline $\bar{\rho}_{0}^{2 *}$ & & \\
\hline $\bar{\rho}_{c}^{2} \dagger$ & & \\
\hline
\end{tabular}

${ }^{*} \bar{\rho}_{0}^{2}=1-\frac{[L(\hat{\beta})-K]}{L(0)}$, where $K$ is the number of parameters in the model (including the constant).

$\dagger \bar{\rho}_{c}^{2}=1-\frac{[L(\hat{\beta})-(K-1)]}{L(c)}$ 
TABLE 2 Empirical Results for Teleworking-related Decisions

\begin{tabular}{|c|c|c|c|c|}
\hline \multirow{2}{*}{ Explanatory Variables } & \multicolumn{2}{|c|}{ Telecommuting Choice } & \multicolumn{2}{|c|}{ Telecommuting Frequency } \\
\hline & Parameters & t-stat & Parameters & t-stat \\
\hline \multicolumn{5}{|l|}{ Individual Socio-demographics } \\
\hline Female & -0.0758 & -0.512 & & \\
\hline Female with presence of children & 0.3583 & 1.882 & & \\
\hline Caucasian or Hispanic & 0.3564 & 1.301 & -0.6003 & -1.295 \\
\hline \multicolumn{5}{|l|}{ Education } \\
\hline Completed undergraduate degree & 0.6570 & 2.475 & & \\
\hline Completed MS degree & 1.0076 & 3.685 & & \\
\hline Completed PhD & 0.8905 & 2.267 & & \\
\hline Income - greater than $\$ 55,000$ & 0.2347 & 1.639 & & \\
\hline \multicolumn{5}{|l|}{ Household Characteristics } \\
\hline \multicolumn{5}{|l|}{ Work-related Variables } \\
\hline Part-time Employment & 0.6081 & 2.946 & 0.8767 & 2.988 \\
\hline \multicolumn{5}{|l|}{ Employer Type } \\
\hline Educational Institution & 0.3728 & 1.965 & 0.2864 & 0.952 \\
\hline State Government & -0.5794 & -1.851 & & \\
\hline Federal Government & 0.5990 & 1.293 & & \\
\hline \multicolumn{5}{|l|}{ Threshold Parameters } \\
\hline Threshold 1 & 1.5451 & 3.548 & -0.6650 & -0.717 \\
\hline Threshold 2 & - & - & -0.5149 & -0.576 \\
\hline Threshold 3 & - & - & -0.1550 & -0.189 \\
\hline Threshold 4 & - & - & 0.4098 & 0.573 \\
\hline Threshold 5 & - & - & 1.3296 & 2.178 \\
\hline Correlation Parameter & \multicolumn{4}{|c|}{$0.673(2.266)$} \\
\hline Number of observations & \multicolumn{4}{|c|}{687} \\
\hline Log-likelihood at equal shares $-L(0)^{*}$ & \multicolumn{4}{|c|}{-623.12} \\
\hline Log-likelihood at sample shares $-L(c)^{\dagger}$ & \multicolumn{4}{|c|}{-385.232} \\
\hline Log-likelihood at convergence $-L(\hat{\beta})$ & \multicolumn{4}{|c|}{-348.918} \\
\hline $\bar{\rho}_{0}^{2} \ddagger$ & \multicolumn{4}{|c|}{0.405} \\
\hline $\bar{\rho}_{c}^{2} \S$ & \multicolumn{4}{|c|}{0.055} \\
\hline
\end{tabular}

* The log-likelihood value at equal shares corresponds to the case where (a) the probability of each individual teleworking is 0.5 , (b) the probability of each of the six teleworking frequency categories for each teleworker in the sample is $1 / 6=0.167$, and (c) the correlation parameter is 0 .

† The log-likelihood value at sample shares corresponds to the case where (a) the probability of each individual teleworking is equal to the share of teleworkers in the sample, (b) the probability of each of the six teleworking frequency categories for each teleworker is equal to the corresponding teleworking frequency sample shares, and (c) the correlation parameter is zero. This is equivalent to the model with only thresholds in the model.

$\ddagger \bar{\rho}_{0}^{2}$ is computed in the same way as the expression in Table 1 (with $K$ being the total number of parameters in the joint model).

$\S \bar{\rho}_{c}^{2}=1-\frac{[L(\hat{\beta})-(K-T)]}{L(c)}$, where $T$ is the total number of thresholds (6 in the current model). 
TABLE 3 Choice Model Results for Flexible Schedule

\begin{tabular}{|c|c|c|}
\hline Explanatory Variable & Parameter & t-statistic \\
\hline \multicolumn{3}{|l|}{ Individual Sociodemographics } \\
\hline Age - less than 25 years & -0.650 & -1.485 \\
\hline Income greater than $\$ 55,000$ & 0.182 & 1.999 \\
\hline Completed under-graduate degree or higher & 0.309 & 2.444 \\
\hline \multicolumn{3}{|l|}{ Household Characteristics } \\
\hline Presence of children less than 5 years & 0.235 & 1.729 \\
\hline \multicolumn{3}{|l|}{ Work-related Variables } \\
\hline Work start time before $9 \mathrm{AM}$ & -0.473 & -3.381 \\
\hline Work place located in CBD or urban area & 0.222 & 2.165 \\
\hline \multicolumn{3}{|l|}{ Threshold Parameters } \\
\hline Threshold 1 & 0.843 & 1.874 \\
\hline Threshold 2 & 1.226 & 2.723 \\
\hline Threshold 3 & 1.631 & 3.613 \\
\hline Threshold 4 & 1.952 & 4.314 \\
\hline Log-likelihood at equal shares $-L(0)^{*}$ & \multicolumn{2}{|c|}{-1057.4} \\
\hline Log-likelihood at sample shares $-L(c)^{\dagger}$ & \multicolumn{2}{|c|}{-981.42} \\
\hline Log-likelihood value at convergence $-L(\hat{\beta})$ & \multicolumn{2}{|c|}{-964.68} \\
\hline $\bar{\rho}_{0}^{2} \ddagger$ & \multicolumn{2}{|c|}{0.078} \\
\hline $\bar{\rho}_{c}^{2} \S$ & \multicolumn{2}{|c|}{0.011} \\
\hline
\end{tabular}

\footnotetext{
* The log-likelihood value at equal shares corresponds to the case where the probability of each ordinal flexibility category for each worker is $1 / 5=0.2$. This model corresponds to the case where only thresholds are included and are constrained to be $-0.842,-0.253,0.253$, and 0.842 .

† The log-likelihood value at sample shares corresponds to the case where the probability of each ordinal flexibility category for each worker is equal to the sample share of that ordinal category. This corresponds to the case where only thresholds are included in the model.

$\ddagger \bar{\rho}_{0}^{2}$ is computed in the same way as in Table 1 .

$\S \bar{\rho}_{c}^{2}$ is computed in the same manner as in Table 2.
} 